\title{
LA SATISFACCIÓN SUBJETIVA DEL ABUELO Y LA ABUELA "CANGURO"
}

\author{
Verónica García Díaz \\ Universidad de Oviedo \\ garciaveronica.fpe@gmail.com \\ Lucía Álvarez-Blanco \\ Universidad de Oviedo \\ Ma $^{\mathrm{a}}$ Teresa Iglesias García \\ Universidad de Oviedo
}

Recepción Artículo: 15 abril 2020

Admisión Evaluación: 17 abril 2020

Informe Evaluador 1: 17 abril 2020

Informe Evaluador 2: 119 abril 2020

Aprobación Publicación: 20 abril 2020

\section{RESUMEN}

Obligado a adaptarse al entramado social que lo rodea, el núcleo familiar actual enfrenta múltiples situaciones sociofamiliares que le hacen ampliar su red de apoyo y dar cabida a los abuelos como cuidadores e, incluso, educadores auxiliares de sus nietos y nietas. Tal implicación por su parte es generalmente considerada fuente de sentimientos positivos, si bien no puede obviarse su posible incompatibilidad con el descanso y disfrute esperados para la etapa evolutiva en la que se encuentran. Al respecto, y por la variedad de circunstancias personales que hoy rodean a abuelos y abuelas, el presente trabajo tiene como objetivo conocerlas e indagar en su grado de satisfacción con ellas. Para ello se ha realizado un estudio ex post facto con una muestra de 350 abuelos y abuelas, a los que se ha aplicado un cuestionario de elaboración propia. Su respuesta revela que la mayoría tiene una situación personal que facilita la asunción de este cometido, y con la que unos y otras están altamente satisfechos. Ser relativamente joven, no trabajar fuera de casa, no tener problemas de salud que impidan su actividad diaria, o disponer de tiempo para sí mismos y aprovecharlo, son algunos factores que evidencian tal bienestar en la muestra consultada. No obstante, cabe aludir también a la otra parte que comparte una visión más negativa de su realidad y que, por ende, hace que surja la ineludible necesidad de apoyar y orientar socioeducativamente a estos abuelos y abuelas.

Palabras clave: abuelos; cuidado de nietos; satisfacción; familia

\section{ABSTRACT}

Subjective satisfaction of grandparents babysitting. The current family circle, forced to get adapted continually to the surrounding social structure, faces numerous social and family situations. This situation makes families broaden their networks of support and include hence grandparents as caregivers and even supplementary educators for their grandchildren. Such involvement of grandparents is generally considered a source of positive 


\section{LA SATISFACCIÓN SUBJETIVA DEL ABUELO Y LA ABUELA "CANGURO"}

feelings. However, it cannot be ignored its potential incompatibility with the expected rest and enjoyment of their current life stage. In that regard, and given the wide range of individual circumstances of contemporary grandparents, this research aims to get to know these circumstances and delve into the satisfaction with them. To this effect, an ex post facto study has been carried out with a sample of 350 grandparents, using an instrument of our own elaboration. Results show that most of the participants have a personal situation that eases the assumption of such tasks, and with which they are highly satisfied. Being relatively young, not working outside the home, being healthy enough to accomplish their everyday activities, or having time for themselves are some of the factors that prove such wellbeing in the sample analyzed. Nonetheless, mention should be made to the other part of the sample, sharing a more negative vision of their reality and arising hence the inescapable necessity to support and guide these grandparents at a social and educational level.

Keywords: grandparents; grandchildren caring; satisfaction; family

\section{INTRODUCCIÓN}

Hoy en día son el abuelo y/o la abuela quienes por la llamada solidaridad intergeneracional asumen, con mayor asiduidad que cualquier otro miembro de la familia extensa, la atención de sus nietos y nietas cuando por diversos motivos, principalmente laborales, sus padres y/o madres no pueden asumirla. Así pues, el abuelo y abuela actual asumen un papel que lleva a que comúnmente se le considere como un elemento provisor del bienestar familiar clave en dicha atención a la infancia, máxime si además se tiene en cuenta que ya no solo abarca funciones de cuidado básico y la tradicional transmisión de valores, sino también otras responsabilidades más exigentes. Según autores como Roa, Herrera y Pulido (2016), en ellos se delegan ciertas funciones educativas que se añaden a esas otras que han venido asumiendo en épocas pasadas, lo que deriva en un protagonismo compartido con el padre y/o madre en torno al correcto desarrollo de sus hijos e hijas (González, De la Fuente y González, 2015).

La mayoría de autores hacen mención a que tal implicación por parte del abuelo y/o abuela favorece la participación e integración familiar y social, al tiempo que repercute positivamente en el tipo de relación que hoy establecen con sus nietos y nietas y en la salud mental del colectivo. Se superan así viejos prejuicios y estereotipos que daban una imagen distorsionada del abuelo y/o abuela como sujetos pasivos, sin posibilidad de implicarse ni de responsabilizarse de tareas como el cuidado de sus nietos y nietas. A tal respecto, autores como Gomila (2005) y González y De la Fuente (2008) hacen hincapié en la contribución del aumento de esperanza de vida, al generar una población mayor cada vez más extensa con mejores condiciones y competencias, capaz de asumir una actitud activa y de apoyo hacia generaciones sucesoras. La Organización Mundial de la Salud (2002), concibe este envejecimiento activo como un proceso por el que se optimiza toda oportunidad de mejorar el bienestar de la persona a medida que envejece.

Siguiendo a Malla (2004), ello abre una puerta a la llamada "generatividad", un concepto que, para Villar et al. (2013, p. 62), parte de la capacidad de la persona "[... ] para desarrollarse y crecer [... ] a partir de la realización de actividades que contribuyen a la mejora de los contextos en los que viven y participan", como es hacer de "canguro" o cuidador auxiliar de sus nietos y nietas.

Tal ejercicio por su parte es casi generalizadamente considerado como fuente de sentimientos positivos. Así, se encuentran numerosas referencias al disfrute del rol, al sentimiento de utilidad, futuro y optimismo que conIleva su activa asunción, al aumento de seguridad en sus propias capacidades, y a la satisfacción y felicidad que ello genera en el abuelo y/o abuela. Autores como Rosenberg \& Mc Cullough (1981), Strawbridge et al. (1997), Triadó et al. $(2009,2014)$, reconocen estos sentimientos como aquellos fundamentalmente asociados al ejercicio de su "abuelidad". Por su parte, Villalba (2002) refuerza esta misma idea al mencionar que el abuelo y/o abuela da sentido a su vida y se siente útil, brindando su tiempo libre al cuidado auxiliar de sus nietos y nietas.

No obstante, la visión más positiva de esta función parece desvanecerse cuando la atención hacia sus nietos y nietas es intensiva en tiempo y lleva a ejercer un rol que generacionalmente no le corresponde y que es incompatible con el disfrute y la permisividad que solía caracterizar el papel antes desempeñado. En efecto, Pinazo y 
Ferrero (2003) hacen alusión a que su desarrollo fuera del ciclo evolutivo normalizado conlleva unos riesgos asociados que pueden suponer una enorme carga para el abuelo y abuela y trastornar la cadencia de vida esperada. Para Villalba (2002), ello puede llevarle a debatirse entre el deseo de apoyar a su familia y el deseo de desarrollar sus propias vidas y proyectos; un choque de intereses que puede generarle sentimientos de pérdida, de frustración 0, como señalan Micolta y Escobar (2010), de irritación por la falta de tiempo para dedicarse a sí mismo.

De la posible aparición de tales sentimientos encontrados, se deriva la realización de este trabajo, cuyo principal objetivo es conocer aquellas circunstancias personales y familiares que hoy rodean al abuelo y/o abuela "canguro", y su grado de satisfacción para con ellas. Al respecto, es de interés prestar atención a ese bienestar (García-Viniegras y González, 2000), que abarca la contemplación de dimensiones como la satisfacción con la vida, el ánimo, la percepción de felicidad y del tiempo (Carmona, 2009). Autores como Puig y Rodríguez (2011) añaden a esto la importancia de la disponibilidad económica, de recursos, de servicios, etc.

Como añadido, varios expertos coinciden en señalar que hay factores propios de la persona que, por su repercusión en la percepción que ésta tiene de su situación y en la forma en que la afronta, exigen atención. De lo contemplado por Bernad (2012), Osuna (2006) y Rico, Serra y Viguer (2001), surge la necesidad de no pasar por alto en el presente trabajo la posible repercusión del género y edad del abuelo y/o abuela y el número de nietos y nietas que tienen a su cuidado.

\section{MÉTODO}

\section{Participantes}

Se ha recogido información de 350 abuelos y abuelas, cuyas principales características se presentan a continuación:

Un 60\% ( $n=210)$ son abuelas y el 40\% ( $n=140)$ restante abuelos. El $99.2 \%$ es de nacionalidad española y residen en algún municipio del centro de Asturias.

Con relación a la edad, una parte sustancial de esta muestra (66.1\%) tiene entre 40 y 64 años, mientras que otro 33.9\% tiene 65 o más.

Con respecto al estado civil de esos abuelos y abuelas, se sabe que el $86 \%$ está casado, el $98.6 \%$ vive en su casa y lo hace acompañada de su esposo y esposa (o pareja) y/o de otros familiares (90.2\%).

En cuanto a los nietos y nietas que cuidan, un $54.9 \%$ indica dos, un $36 \%$ uno y un $9.1 \%$ tres o más. Sobre sus nietos y nietas, se recoge que 213 son chicos (60.9\%) y 137 chicas (39.1\%), principalmente de entre uno y diez años (84.9\%).

\section{Instrumento}

Tras la revisión de la literatura correspondiente, no se encontró un instrumento validado que permitiera obtener la información esperada en este trabajo, por lo que se decidió construir un cuestionario propio a tal efecto. Se obtuvo así un cuestionario de 10 ítems con respuesta en escala Likert de cuatro alternativas: 1=nunca o no,

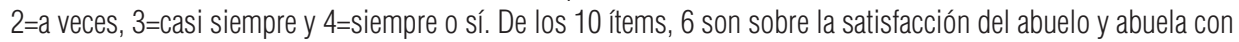
su situación actual y 4 sobre sus recursos y sus apoyos económicos.

Se pregunta también por factores como el género y edad del abuelo y abuela y el número de nietos y nietas que cuidan, pudiendo conocer así la diversidad de la muestra y analizar diferencialmente dichos bloques de contenido.

\section{Procedimiento}

Dado que no existía ningún registro conocido que indique el número de abuelos y abuelas que asumen estas responsabilidades en Asturias, se empleó un muestreo no probabilístico. Concretamente, se ha utilizado un muestreo no probabilístico de "bola de nieve" (snowball sampling) (Goodman, 1961), con el que se invitó a participar a todos aquellos abuelos y abuelas "canguro" que quisieran hacerlo. Estos distribuyeron copias a otros de su entorno y estos a su vez a otros hasta obtener el número necesario. 


\section{LA SATISFACCIÓN SUBJETIVA DEL ABUELO Y LA ABUELA "CANGURO"}

\section{Análisis de datos}

Los datos cuantitativos obtenidos de la aplicación del cuestionario se han analizado con el programa estadístico SPSS versión 22.0.

Una vez depurada la base de datos, se han utilizado técnicas univariadas de tipo descriptivo: frecuencias, porcentajes, medias y desviaciones típicas y de comparaciones entre grupos. Para efectuar tal análisis comparativo, se toman en consideración variables dicotómicas como el género o la edad del abuelo y abuela (categorizada en dos opciones: de 40 a 64 años y de 65 o más), calculándose la t de Student para muestras independientes. En cada análisis se comprobó la condición de homogeneidad de varianzas con la prueba de Levene y el estadístico F. Por su parte, el contraste con variables politómicas se realizó a través del ANOVA de un factor como es el caso del número de nietos y nietas que cuidan. En cada análisis también se tuvo en cuenta la condición de homogeneidad de varianzas a través de la prueba de Levene y del estadístico $\mathrm{F}$, estudiando la diferencia estadística entre parejas de grupos con el método de Scheffé. Si no se cumplía la igualdad de varianzas, se utilizó el estadístico de Welch como alternativa robusta al ANOVA. Seguidamente, se realizó un análisis de discrepancias estadísticas utilizando el método de Games-Howell. El resultado de estos contrastes se consideró estadísticamente significativo a partir de una probabilidad <.05. Cuando con la t se detectaron diferencias se calculó el tamaño del efecto con la d de Cohen considerándose bajo $(0<\mathrm{d}<.20)$, medio $(.20<\mathrm{d}<.50)$ y alto $(\mathrm{d}>.50)($ Cohen, 1988$)$.

\section{RESULTADOS}

En el presente apartado, se ofrecen los resultados obtenidos de la aplicación del cuestionario, siguiendo el orden de análisis indicados en el apartado correspondiente.

Satisfacción de los abuelos/as con su situación actual

Los resultados descriptivos univariados, indican que la gran mayoría de abuelos y abuelas consultados (83\%, n=288) siempre 0 casi siempre se sienten felices y satisfechos con su situación actual, mientras que solo un $17 \%(n=59)$ indica lo contrario (media=3.38; DT=.78).

Cuando se trata de analizar si perciben que están en sus mejores años de vida, se obtiene un porcentaje parecido entre quienes nunca o casi nunca piensan esto $(51.3 \%, n=175)$ y quienes lo piensan siempre 0 casi siempre (48.7\%, n=166). Al respecto, es el enfoque pesimista el que predomina (media=2.62; DT=1.01).

Esta reñida percepción, podría estar condicionada por su salud. Sin embargo, el 86.4\% ( $n=286$ ) señala que nunca o casi nunca tiene problemas al respecto. El 13.6\% ( $n=45$ ) indica que sí los sufre (media=1.64; DT=.925).

El 93.8\% ( $n=315)$ alude a que nunca o casi nunca suele sentirse desanimado y triste, dándose un porcentaje minoritario $(6.3 \%, \mathrm{n}=21)$ que menciona sentirse así siempre o casi siempre (media=1.69; DT=.70).

Sobre la disponibilidad de tiempo personal, un $66.1 \%$ ( $n=224)$ reconoce que siempre 0 casi siempre tiene el necesario para dedicarse a sí mismo y a hacer lo que le satisface. No obstante, el 33.9\% ( $n=115)$ considera lo contrario (media=3.07; $\mathrm{DT}=.94)$. El 51.6\% ( $n=177$ ) siempre 0 casi siempre opta por realizar actividades culturales y de ocio en su tiempo libre, frente al 48.4\% ( $n=166)$ que nunca o casi nunca lo hace (media=2.74; $D T=1.10)$.

Los resultados obtenidos a este respecto por género, revelan que la abuela, en comparación con el abuelo, es quien se siente menos conforme con su situación (t (347)=-2.414; $p=.016 ; d=-.27$ ), menos animada ( $t$ (336)=3.284; $p=.001 ; d=.37$ ) y con escaso tiempo para sí misma (t (339)=-2.223; $p=.027 ; d=-.24$ ).

Por edad, quienes tienen entre 40 y 64 años tienden a estar más desanimados que aquellos con 650 más (t (334)=3.291; $p=.001 ; d=.39$ ) y sin tiempo para sí mismos (t (337)=-3.970; $p=.000 ; d=-.46)$.

Para el contraste por número de nietos y nietas que cuidan (uno, dos y tres o más de tres) se ha calculado ANOVA de un factor, detectándose discrepancias relevantes para la variable "Pienso que estoy en los mejores años de mi vida" entre abuelos y abuelas que cuidan tres o más nietos y nietas y quienes lo hacen de uno 0 dos. Concretamente, y tal como se recoge en la Tabla 1, es el primer grupo el que menor optimismo muestra en este sentido. 
Tabla 1. Diferencias estadísticas significativas sobre la satisfacción en función del número de nietos y nietas que cuidan

\begin{tabular}{llccc}
\hline \multicolumn{1}{c}{ Variable } & Welch & $\mathrm{p}$ & $\mathrm{p}$ Games-Howell \\
\hline $\begin{array}{l}\text { 02. Pienso que estoy en los mejores años de } \\
\text { mi vida }\end{array}$ & 1 & 3.802 & 026 & $.037(3<1)$ \\
& 2 & & & $.027(3<2)$ \\
\hline
\end{tabular}

\section{1: cuida a un nieto y nieta, 2 : a dos, 3 : a tres o más}

\section{Recursos y apoyos económicos del abuelo/a}

El 93.3\% ( $n=319$ ) siempre o casi siempre dispone de recursos económicos para cubrir sus necesidades, si bien el $6.7 \%$ ( $n=23$ ) restante reconoce que nunca o casi nunca posee lo que necesita (media=3.73; DT=.64).

La percepción de disponer -o no- de recursos económicos suficientes puede estar condicionada por la necesidad de estos abuelos y abuelas de apoyar económicamente a otros familiares. Sin embargo, en esta muestra se ha encontrado que el $86.1 \%(n=278)$ nunca 0 casi nunca se ve afectado por esta situación, frente al $13.9 \%$ ( $n$ $=45)$ que indica estarlo (media $=1,42 ; \mathrm{DT}=1,023)$.

Otro aspecto de relevancia a considerar en relación a recursos y apoyos, es si estos abuelos y abuelas tienen - o no- familiares dependientes a su cargo por temas de salud. La mayoría $(87.1 \%, n=291)$ ha indicado que no se encuentra en tal situación, aunque hay un 12.9\% (n=43) que señala que sí (media=1.45; DT=1.00).

Por su parte, cuando se pregunta si reciben algún apoyo de Servicios Sociales, un porcentaje dominante $(94.4 \%, n=289)$ señala que nunca 0 casi nunca se ha visto en esta necesidad. El 5.6\% $(n=17)$ restante indica que sí (media=1.16; DT=.65).

No se encuentran diferencias por género en torno a recursos económicos ni en el cargo que estos abuelos y abuelas tienen para con otros familiares. No obstante, en lo relativo a ese cargo, la edad sí arroja discrepancias, de modo que quienes aún tienen entre 40 y 64 años asumen mayores responsabilidades para con personas dependientes por razón económica (t (321)=2.496; $p=.013 ; d=.28$ ) y salud (t (332)=4.696; $p=.000 ; d=.49$ ).

\section{DISCUSIÓN Y CONCLUSIONES}

Con el presente trabajo se ahonda en la diversidad de circunstancias personales y familiares que rodean al abuelo y/o abuela "canguro", así como en la satisfacción con que perciben su situación actual, su edad o etapa vital, la salud 0 el disfrute de su tiempo.

Así, abuelos y abuelas encuestados se sienten felices y satisfechos con su vida, lo que hace que valoren de un modo optimista su realidad y dejen a un lado sentimientos negativos, de desánimo y tristeza. No obstante, no puede obviarse al porcentaje que dice no sentirse tan conforme con la etapa vital en la que se encuentra. Unido a ello, reconocen no tener problemas de salud física o mental que impidan su actividad diaria. De igual modo, dejan constancia de su tiempo, del aprovechamiento del mismo, de su activa disposición para ello y para la participación en actividades de su interés y para su disfrute personal.

Por ello, y al respecto de dichas circunstancias, se ha considerado oportuno ahondar no sólo en la vitalidad del colectivo, que fruto de cambios como el incremento de la esperanza y la calidad de vida, condiciones físicas y mentales, etc., parece ser de lo más activa, idea reconocida por autores como Limón y Berzosa (2006), Limón y Ortega (2011) o Pinazo (2012a, 2012b), sino también en aspectos relativos a la valoración que éste tiene de su situación. La mayoría de autores citados y otros, como Luján y González (2013) o Miralles (2010), evidencian la misma satisfacción atisbada en este estudio.

Como añadido, parece que su situación económica es adecuada y que facilita, de algún modo, la percepción que tienen en torno a todo lo previo; no habiendo recibido mayoritariamente apoyo de Servicios Sociales, ni teniendo familiares a su cuidado, ya sea por motivos económicos o por salud. 


\section{LA SATISFACCIÓN SUBJETIVA DEL ABUELO Y LA ABUELA "CANGURO"}

La totalidad de estudios revisados, incluyen el género como un factor altamente contribuyente en la aparición de ciertas diferencias. Lo recogido por Casas y Aymerich (2005) o León, Rojas y Campos (2011) va en esta línea, pues indican que es la mujer quien tiene una valoración más negativa que el varón. Ello quizá pueda deberse a la tradicional carga que ésta aún asume para con la familia, complicando su actividad diaria y, como se ha visto, también la disponibilidad y el disfrute de su tiempo libre.

La misma atención prestada a la variable género, se le concede también a la edad. Así, y teniendo en cuenta los resultados, no sólo se corrobora que en función de sus años se dan ciertas discrepancias concluyentes en lo referente a la satisfacción del colectivo, sino que también se demuestra que la aún estereotipada visión del abuelo y abuela como un sujeto pasivo, sin posibilidad siquiera de implicarse ni responsabilizarse del cuidado de sus nietos y nietas, está quedando obsoleta. Diener \& Shu (1998) comparten esta misma idea.

Ocurre lo mismo con el disfrute de su propio tiempo, pues aquellos más jóvenes consideran, con mayor frecuencia que aquellos que tienen una edad más avanzada, que éste es bastante escaso. Ello, concuerda perfectamente con el resultado de que aquellos abuelos y abuelas que tienen entre 40 y 64 años asumen mayores responsabilidades en la manutención y cuidado de familiares dependientes. En este sentido, lo obtenido va en total coherencia con lo indicado por Villar et al. (2006), que hablan sobre el aumento del tiempo dedicado al ocio y descanso en la etapa de la vejez y la disminución del dedicado a las obligaciones cotidianas.

Sobre la variable "Pienso que estoy en los mejores años de mi vida", se detectan diferencias relevantes en torno al número de nietos y nietas a su cuidado, pues parece ser que cuantos más, peor visión tienen de la edad o etapa evolutiva en la que se encuentran. Lo obtenido coincide con lo mencionado en la introducción de este artículo de la intensidad del cuidado y sus posibles efectos negativos.

Así, y partiendo de lo aquí concluido, surge la ineludible necesidad de apoyo a la parte de la muestra que tiene una valoración más negativa de su realidad. Puesto que parecen ser quienes más atención precisan al observarse en ellos mayor negatividad, quizá sea de interés diseñar líneas de actuación que fomenten la adquisición de estrategias para una óptima organización diaria, que redunde positivamente en la satisfacción del abuelo y, aún más, de la abuela relativamente joven.

\section{REFERENCIAS BIBLIOGRÁFICAS}

Bernad, J. A. (2012). De profesión, abuelo. Ser abuelo hoy (... con vocación y fundamento). Barcelona: Editorial Graó.

Carmona, S. E. (2009). El bienestar personal en el envejecimiento. Revista de Ciencias Sociales de la Universidad Iberoamericana, 7, 48-65.

Casas, F. y Aymerich, M. (2005). Calidad de vida de las personas mayores. En S. Pinazo y M. Sánchez (Dir.), Gerontología. Actualizaciones, innovación y propuestas (pp. 115-143). Madrid: Pearson Prentice Hall.

Cohen, J. (1988). Statistical power analysis for the behavioural science. (2nd ed.). Hillsdale, NJ: Lawrence Erlbaum Associates.

Diener, E. \& Suh, E. (1998). Age and subjective well-being: an international analysis. Annual Review of Gerontology \& Geriatrics, 17, 24-41.

García-Viniegras, C. R. V. y González, I. (2000). La categoría bienestar psicológico. Su relación con otras categorías sociales. Revista Cubana de Medicina General Integral, 16(6), 586-592.

Gomila, M. (2005). Las relaciones intergeneracionales en el marco de la familia contemporánea: cambios y continuidades en transición hacia una nueva concepción de la familia. Historia contemporánea, 31, 505-542.

González, J. y De la Fuente, R. (2008). Relevancia psico-socio-educativa de las relaciones generacionales abueI0-nieto. Revista Española de Pedagogía, 239, 103-118.

González, J., De la Fuente, R. y González, J. (2015). En S. Rivas (Coord.), Generaciones conectadas. Beneficios educativos derivados de la relación entre nietos y abuelos (pp. 105-120). Madrid: Ediciones Pirámide.

Goodman, L. A. (1961). Snowball Sampling. Annals of Mathematical Statistics, 32, 148-170. 
León, D, Rojas, M. y Campos, F (2011). Guía calidad de vida en la vejez. Herramientas para vivir más y mejor. Santiago, Chile: Pontificia Universidad Católica de Chile.

Limón, M. R. y Berzosa, G. (2006). Siglo XXI, Envejecimiento y solidaridad. Revista Galega do Ensino, 14(48), 351-375.

Limón, M. R., y Ortega, M. C. (2011). Envejecimiento activo y mejora de la calidad de vida en adultos mayores. Revista de Psicología y Educación, 6, 225-238.

Luján, I. y González, E. (2013). Apoyo de la familia en el tránsito a la jubilación. INFAD Revista de Psicología. International Journal of Developmental and Educational Psychology, 2(1), 287-296.

Malla, R. (2004). Contexto familiar y desarrollo psicológico durante la adultez y la tercera edad. En E. Arranz (Coord.), Familia y desarrollo psicológico (pp. 124-145). Madrid: Pearson Prentice Hall.

Micolta, A. y Escobar, M. C. (2010). Si las abuelas se disponen a cuidar, madres y padres pueden emigrar. Revista Venezolana de Estudios de la Mujer, 15(35), 91-115.

Miralles, I. (2010). Vejez productiva: el reconocimiento de las personas mayores como un recurso indispensable en la sociedad. Kairos: Revista de Temas sociales, 26.

Organización Mundial de la Salud (2002). Envejecimiento activo: un marco político. Revista Española de Geriatría y Gerontología, 37(S2), 74-105.

Osuna, M. J. (2006). Relaciones familiares en la vejez: vínculos de los abuelos y de las abuelas con sus nietos y nietas en la infancia. Revista Multidisciplinar de Gerontología, 16(1), 16-25.

Pinazo, S. (2012a). Envejecimiento activo y solidaridad intergeneracional. En M. G. Pérez, Envejecimiento activo y solidaridad intergeneracional: claves para un envejecimiento activo (pp. 1-20). Universidad Nacional de Educación a Distancia, UNED.

Pinazo, S. (2012b). Las personas mayores proveedoras de conocimiento y cuidados. El papel de los programas intergeneracionales. Educación Social: Revista de Intervención Socioeducativa, 51, 45-66.

Pinazo, S. y Ferrero, C. (2003). Impacto psicosocial del acogimiento familiar en familia extensa: el caso de las abuelas y los abuelos acogedores. Revista Multidisciplinar de Gerontología, 13(2), 89-101.

Puig, M. y Rodríguez, N. (2011). Calidad de vida, felicidad y satisfacción con la vida en un grupo de mayores en una zona rural. Nursing, 29(9), 57-59.

Rico, C., Serra, E. y Viguer, P. (2001). Abuelos y nietos: abuelo preferido, abuelo útil. Madrid: Pirámide.

Roa, J. M., Herrera, F., y Pulido, F. (2016). Valor educativo de los abuelos. En J. L. Castejón, Psicología y Educación: presente y futuro (pp. 2056-2064). ACIPE.

Rosenberg, M. \& Mc Cullough, B. C. (1981). Mattering: inferred significance and mental health among adolescents. In R. Simmons (Ed.), Research in community and mental health (pp. 163-182). Greenwich: JAI Press.

Strawbridge, W., Wallhagen, M., Shema, S., \& Kaplan, G. (1997). New burdens or more of the same? Comparing grandparent, spouse an adult-child caregivers. The Gerontologist, 37(4), 505-510.

Triadó, C., Villar, F., Celdrán, M. \& Solé, C. (2014). Grandparents who provide auxiliary care for their grandchildren: satisfaction, difficulties, and impact on their health and wellbeing. Journal of Intergenerational Relationships, 12(2), 113-127.

Triadó, C., Villar, F., Solé, C., Celdrán, M., Pinazo, S., y Conde, L. (2009). Los abuelos/as cuidadores de sus nietos: percepción de ayudas recibidas, conductas problemáticas de los nietos y satisfacción con el rol. INFAD Revista de Psicología. International Journal of Developmental and Education Psychology, 3(1), 497-506.

Villalba, C. (2002). Abuelas cuidadoras: una aportación para el trabajo social. Valencia: Tirant lo Blanch.

Villar, F., Celdrán, M., Fabà, J. y Serrat, R. (2013). La generatividad en la vejez: extensión y perfil de las actividades generativas en una muestra representativa de personas mayores españolas. Revista Iberoamericana de Gerontología, 1(1), 61-79.

Villar, F., Triadó, C., Solé, C. y Osuna, M. J. (2006). Patrones de actividad cotidiana en personas mayores: ¿es 10 que dicen hacer lo que desearían hacer? Psicothema, 18(1), 149-155. 
